\title{
EPICS, the exoplanet imager for the E-ELT
}

\author{
M. Kasper ${ }^{1, \text { a }}$, J.-L. Beuzit ${ }^{2}$, C. Verinaud ${ }^{2}$, P. Baudoz ${ }^{3}$, A. Boccaletti ${ }^{3}$, R. Gratton ${ }^{4}$, C. Keller ${ }^{5}$, F.

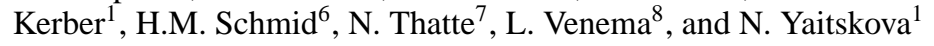 \\ 1 ESO, Karl-Schwarzschild-Str. 2, 85748 Garching, Germany \\ 2 LAOG, Rue de la Piscine 414, 38400 Saint-Martin d'Hères, France \\ 3 LESIA, Place Jules Janssen 5, 92195 Meudon Cedex, France \\ 4 OAPD, Vicolo dell'Osservatorio 5, 35122 Padova, Italy \\ 5 University of Utrecht, Heidelberglaan 100, 3584 CX Utrecht, The Netherlands \\ 6 ETHZ, Wolfgang-Pauli-Str. 27, 8093 Zuerich, Switzerland \\ 7 University of Oxford, Keble Road, Oxford, OX1 3RH, England \\ 8 ASTRON, Oude Hoogeveensedijk 4, 7991 PD Dwingeloo, The Netherlands
}

\begin{abstract}
Very soon, dedicated instruments developments at large telescopes (SPHERE for the VLT, GPI for Gemini) are about to discover and explore self-luminous giant planets by direct imaging and spectroscopy in significant numbers. The next generation of 30m-40m ground-based telescopes, the Extremely Large Telescopes (ELTs), have the potential to dramatically enlarge the discovery space towards older giant planets seen in reflected light and ultimately even a small number of rocky planets. EPICS is a proposed instrument for the European ELT, dedicated to the detection and characterization of expolanets by direct imaging and spectroscopy. EPICS is currently mid-way through a phase-A study carried out by a large European consortium which - by simulations and demonstration experiments - will investigate state-of-the-art diffraction and speckle suppression techniques to deliver highest contrasts. The final result of the study early 2010 will be a conceptual design and a development plan for the instrument. We will present the EPICS concept including the performance analysis and first results from prototyping experiments and discuss the main challenges and science capabilities of EPICS.
\end{abstract}

\section{Introduction}

\subsection{The EPICS phase-A study for the E-ELT}

The Exo-Planet Imaging Camera and Spectrograph (EPICS) is an instrument project for the direct imaging and characterization of extra-solar planets with the European ELT (E-ELT). EPICS will be optimized for observations in the visible and the near-IR and will have photometric, spectroscopic and polarimetric capabilities.

The E-ELT is currently going through a phase-B study which will include an instrumentation plan with the list of first generation instruments. The highest priority scientific objectives (which include Exoplanets) will dictate the choice of the first generation instruments. The EPICS phase-A study is one of these E-ELT instrument studies and has been kicked off in October 2007. Among the goals of EPICS phase-A study, requirements to the telescope and the associated site should be clearly addressed and feedback should be provided to the E-ELT Design Reference Missions. The final study report will include the calculated scientific capabilities, cost, FTE effort and a construction schedule. It will be the basis for the technical specifications and statement of work of the construction contract, should the priority and feasibility of EPICS be confirmed.

The EPICS phase-A study is carried out by a large European consortium led by ESO and LAOG as PI and Co-PI institutes, and is funded in parts by ESO and the European Framework Programme 7 (FP7).

\footnotetext{
a e-mail: mkasper@eso.org
} 


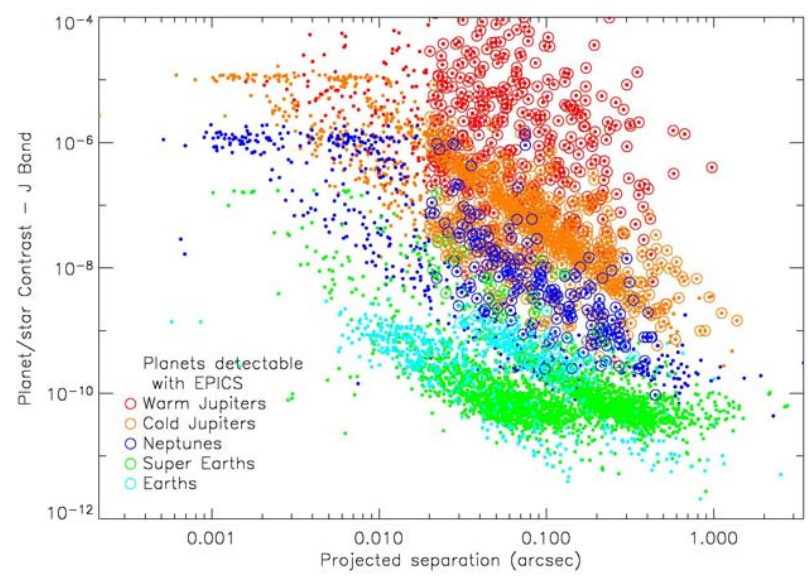

Fig. 1. Monte Carlo simulations of exoplanet detection rates with EPICS.

\subsection{Science goals and TLR}

EPICS will be optimized, and trade-offs made, focusing on the following prominent exoplanet science cases:

1. Detection of young self-luminous gas giants in star forming regions or young associations with the goal to determine initial frequency and mass distribution of giant planets and to study planet formation.

2. Detection and characterization of mature gas giants at orbital distances between 5 and 10 AU in the solar neighborhood $(<20 \mathrm{pc})$ with the goal to determine frequency and mass distribution of giant planets not easily accessible to radial velocity, astrometric or photometric techniques.

3. Imaging and spectral characterization of warm or young Jupiters that have been previously discovered by radial velocity searches or direct imaging with smaller telescopes with the goal is to understand giant planets' atmospheric composition and structure. Some tens of currently known targets are readily observable with EPICS, and many more may be known from upcoming observations with e.g. GAIA or SPHERE.

4. Detection and 1st order characterization of Neptune mass planets and massive rocky planets around nearby stars $(<10 \mathrm{pc})$ with the ultimate goal of detecting such planets located in the habitable zone (for late type stars and very nearby systems $<4 \mathrm{pc}$ ).

In order to achieve these science goals, EPICS will have to reach contrast levels of $10^{-8}$ at 30 mas and $10^{-9}$ between 100 and 300 mas for stars of magnitude 7 in I-band and brighter.

Considering the photon noise limit corresponding to $4 \mathrm{~h}$ of integration time, $10 \%$ light throughput, simulated AO performance for the given star magnitude and a spectral deconvolution data reduction scheme, the number of expected planet detections with EPICS as a function of mass for our combined sample of young stars in star-forming regions and nearby stars has been calculated and is shown in figure 1. These estimates are based on Monte Carlo simulations, where orbital and mass distributions of planets were derived from theoretical models developed by (6) which have been normalized to actual detection rates derived from radial velocity search programs. The plot shows one such realization of these Monte Carlo simulation where the dots denote the modeled planet population surrounded by open circles if detected by EPICS. Some planets are too faint to be discovered although their contrast to the parent star is better than the EPICS contrast TLR, while there are very few planets that are bright enough to be detected if EPICS would deliver an even better instrumental contrast. In total, EPICS will be able to directly image hundreds of gaint panets, dozens of Neptunes and still a fair number of rocky planets for the considered target sample. 


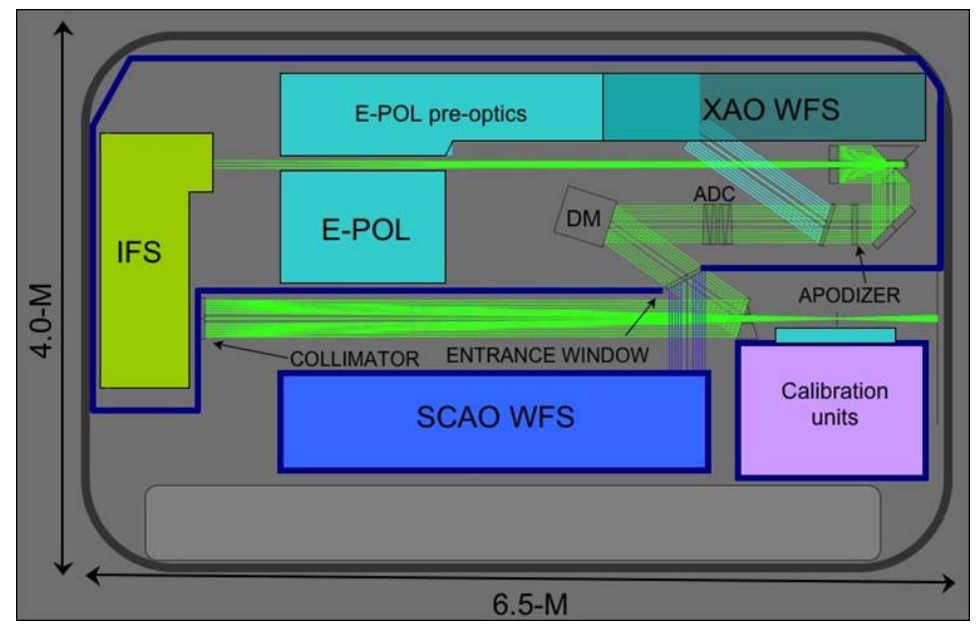

Fig. 2. Conceptual design of EPICS.

\section{Conceptual Design}

Figure 2 outlines the basic concept of EPICS. This concept largely follows the standard approach to the high-contrast imaging problem, where wavefront and diffraction control reduce quasi-static PSF residuals to far below (typically to $10^{-6}$ ) the Airy pattern's peak intensity. Quasi-static wavefront errors are measured by the science instruments using either phase-diversity (7)or speckle nulling. These PSF residuals are then calibrated and largely removed by data analysis techniques discriminating between speckles and potential faint companions based on their chromaticity (spectral deconvolution), polarization properties (differential polarimetry), temporal-spatial behavior (speckle smearing, angular differential imaging), or their coherence with the central source (self-coherent camera). This calibration step can further reduce PSF residuals by typically a factor $10^{3}$, such that the ultimately desired contrast level of around $10^{-9}$ is reached on bright sources.

A gray beam-splitter sends $10 \%$ of the light to a first stage SCAO WFS which commands the telescope M4 and reduces turbulent aberrations to levels that the internal 210x210 actuator DM (20 $\mathrm{cm}$ aperture pitch) can cope with. EPICS will use a 3-glass ADC in the common path leaving very low chromatic residuals ( $<1$ mas PTV by design) over the operation bandwidth between $600 \mathrm{~nm}$ and $1700 \mathrm{~nm}$. By dogin this, we avoid rotating components in the non-common path and hence temporally variable aberrations not seen by the WFS. A dichroic then reflects the wavelengths shorter than 950 $\mathrm{nm}$ to the optical arm and transmits the NIR.

The NIR arm hosts an apodizer and zoom optics to provide an f/140 focus on the input of the IFS. The whole optical trail up to the IFS input focus consists of optical components that are located in or close to the pupil plane. This is to avoid mixing of phase into amplitude errors that would affect speckle chromaticity and limit the efficiency of spectral deconvolution. Figure 3 shows spectra of speckles created by phase aberrations placed in the pupil plane (left) or at 10 Talbot lengths away from it (right). While the speckle intensity smoothly drops inversely proportional to wavelength squared and hence perfectly fit a low-order polynomial in the first case, intensity ripples around the the low order polynomial that would not be removed by spectral deconvolution can be seen in the second case. Following this philosophy, diffraction suppression is achieved by amplitude apodization only rather than a coronagraph which would require a mask or some sort of re-imaging optics near the image plane. In order to damp the stellar light and to reduce problems with ghosting, stray light or detector saturation, a mask will be placed in the entrance image plane of the IFS.

Entering the optical arm, the light is immediately split again with wavelengths longer than 800 $\mathrm{nm}$ entering the WFS and shorter wavelengths being directed to differential optical polarimeter EPOL. EPOL is intrinsically achromatic, so speckle chromaticity isn't an issue and a coronagraph can efficiently be used. The EPICS optical design minimizes the number of reflective optics at large inclination 
First conference on Adaptive Optics for Extremely Large Telescopes

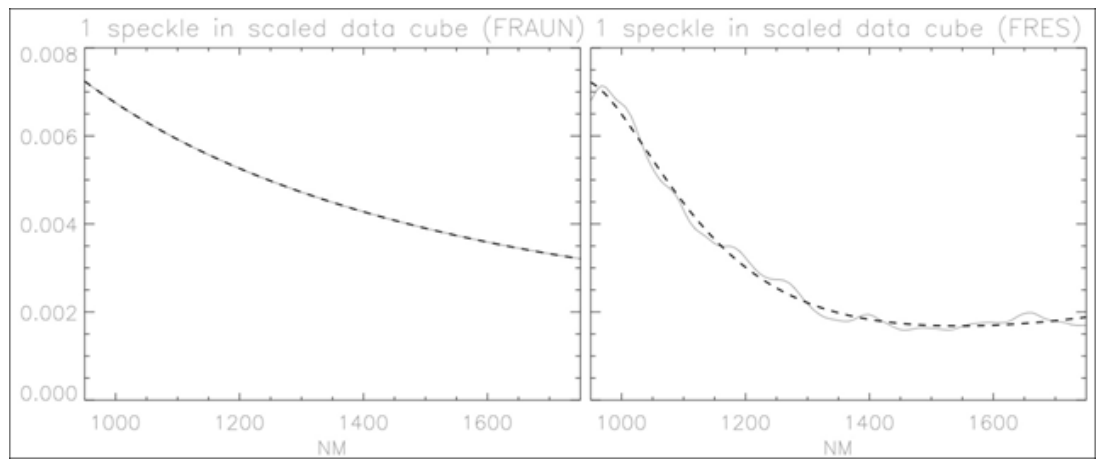

Fig. 3. Spectra of speckles created by phase aberrations close to (left) and away from (right) the pupil plane.

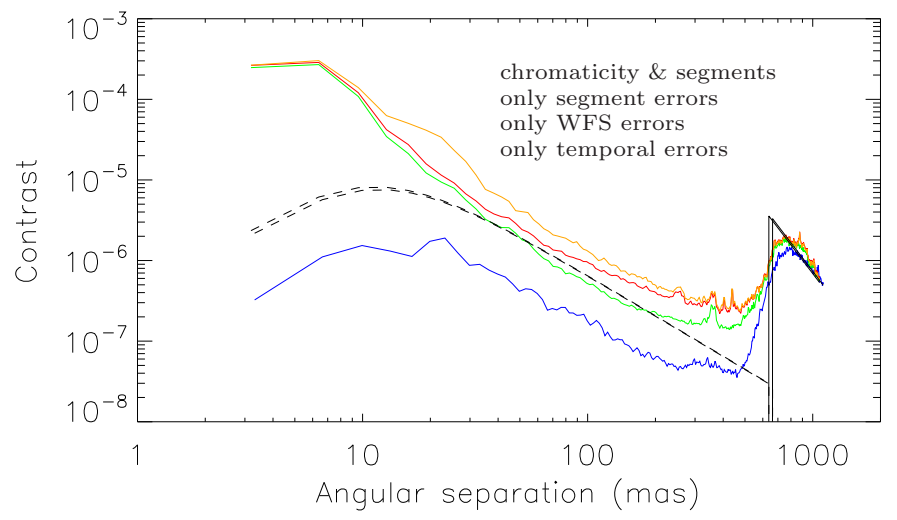

Fig. 4. Radially averaged contrasts of EPICS XAO at a wavelength of $1.3 \mu \mathrm{m}$.

angles introducing instrumental polarization and foresees calibration devices for those that cannot be avoided such as the telescope's M5 mirror.

The major design guidelines (optics close to pupil plane and normal incidence) are hardly compatible with a K-mirror for optical de-rotation, such that EPICS does not implement one. However, field de-rotation of the IFS will be needed for its pseudo long-slit high spectral resolution mode, and pupil de-rotation would be needed for efficient angular differential imaging (ADI). Both goals could be achieved by having a mechanism able to rotate the complete EPICS instrument. While this solution would be a major cost driver for the instrument and the potential benefits of ADI are uncertain, EPICS will de-rotate the IFS only, if needed to stabilize the field.

\section{XAO}

The EPICS XAO system will feature two cascaded and independent AO loops. The first loop is a classical single conjugate (SCAO) system using the E-ELT wavefront correction, M4 and M5, for low-order and tip/tilt correction. The baseline SCAO WF sensor is the modified optical differentiation sensor (MODS) described by (3). It has a sufficient dynamical range and is not propagating excessively noise on the high spatial frequencies. The XAO sensor is a non-modulated roof sensor (RS). The 1st and 2nd stage WFSs have a sampling of $80 \times 80$ and 200x200 subapertures, respectively, and sensing is done at $0.825 \mu \mathrm{m}$ in both cases.

It can be seen that the WFS error reduces the contrast from about $5 \times 10^{-8}$ to $2 \times 10^{-7}$ at 200 to 600 mas and more than this at smaller angular separations ( $<10$ mas). Segment mis-figure and chromaticity 

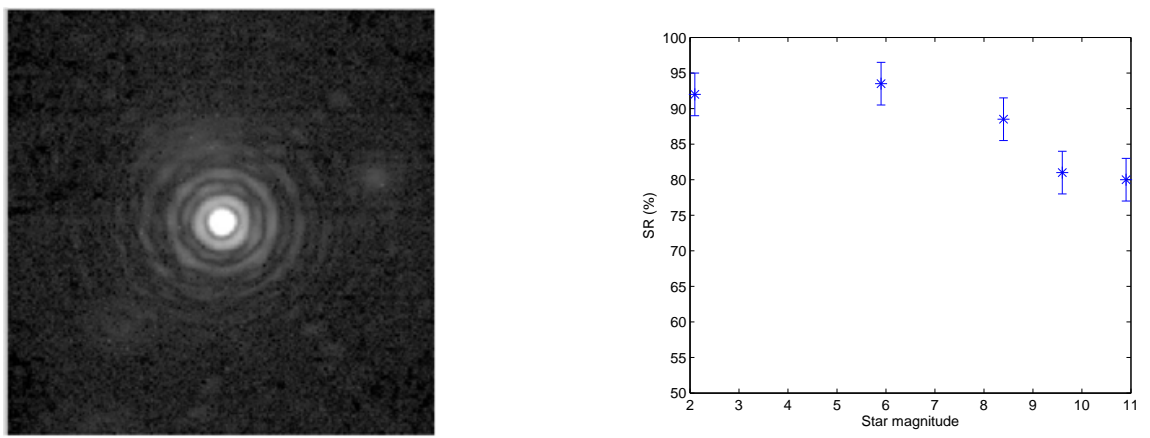

Fig. 5. Left: XAO corrected PSF with H-band SR > 90\%, Right: SR performance as a function of the star magnitude

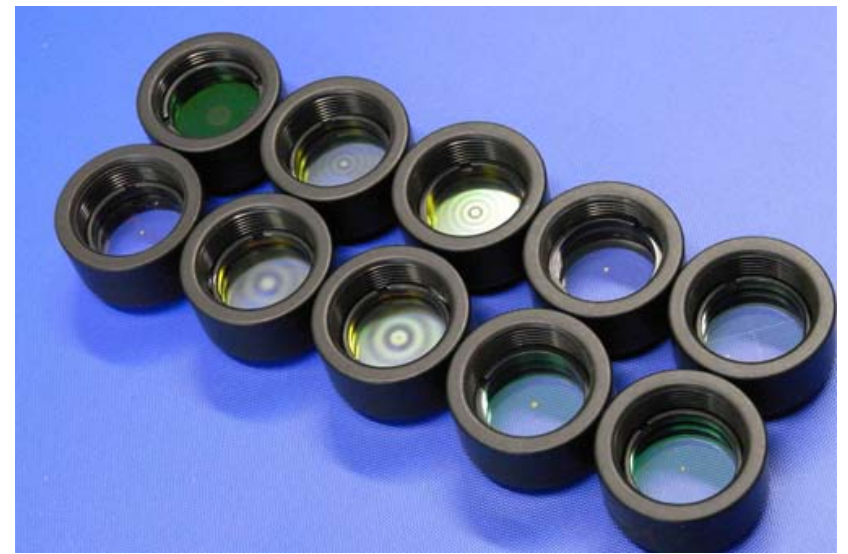

Fig. 6. Various coronagraphic masks and apodizers manufactured for and tested for EPICS.

effect further reduce the contrast by an additional factors of 2 between 100 to 400 mas and 10 to 100 mas, respectively.

\section{Enabling technology and concepts}

Various crucial aspects of the EPICS concept are being investigated by experiment during the EPICS phase-A. One of the key tools is ESO's high order testbench (HOT) which has been developed in collaboration with Durham University and Arcetri and was supported by the European Framework Programme 6. HOT features atmospheric turbulence generations, XAO using a 60-element bimorph large stroke DM and a 32x32 MEMS DM, and L3-camera based Pyramid or spatially filtered ShackHartmann sensors. HOT further implements an NIR corongraphic imaging arm. With HOT, we were able to demonstrate that XAO can produce very well corrected images with $\mathrm{H}$-band Strehl ratios above 90\% as shown in Fig.5 (1) and to demonstrate coronagraphic contrast performance as predicted by simulations (4). Fig.6 shows some of the various coronagraphic masks and apodizers that were evaluated with HOT (5).

Apart from XAO and coronagraphy the efficient calibration and correction of quasi-static speckles is of paramount interest and a number of strategies tackling this problem from different sides are being evaluated. The identification and removal of quasi-static speckles by focal plane wavefront sensing techniques (such as phase diversity and speckle nulling) are being tested on a dedicated test bench built at LAOG (FFREE) and supported by the European framework programme 7 (FP7). An alternative 


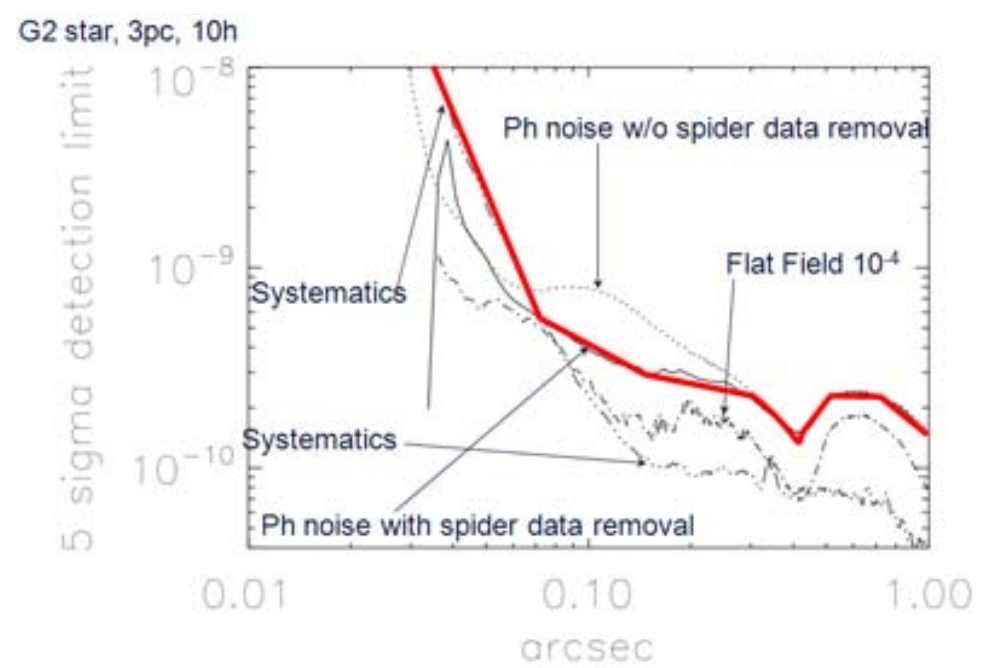

Fig. 7. Contrast performance of EPICS for a very bright star calculated with an end-2-end model and analytic $\mathrm{XAO}$ correction.

technique exploiting the coherence of speckles in contrast to a planetary companion is provided by the Self-Coherent camera (SCC, (2)) that is being evaluated at LESIA. Finally, aspects of the calibration of quasi-static speckles either by an IFS through spectral deconvolution or by an imaging polarimeter through differential polarimetry are being investigated at Padova Observatory, Oxford University, ETHZ, ASTRON and Utrecht University again supported by FP7.

In the longer term, the key enabling technologies for EPICS will be the high-order deformable mirror with about 30.000 actuators including its drive electronics and the real-time computer with requirements far beyond what is achievable with current approaches.

\section{Performance Analysis}

In order to design EPICS and evaluate its performance considering as many as possible real-life error sources, we developed an end-2-end model of the instrument called PESCA (Parallel EPICS Simulation Codes and Applications). PESCA models the complete chain from atmospheric turbulence including AO error terms, over the telescope with its segmented primary and large M2 support structure, to the instrument with quasi-static aberrations and diffraction suppression systems and spectral deconvolution using a Fresnel propagation code. Fig.7 shows a resulting contrast curve for a very bright star demonstrating that the EPICS concept is pushing the systematic limits below the photon noise level and achieves the contrast levels of the order $10^{-9}$ at small angular separations required for the efficient detection of illuminated extrasolar planets in the NIR.

\section{References}

1. Aller-Carpentier, E. et al. 2009, AO4ELT conf., Eds: Y. Clénet, J.M. Conan, T. Fusco, G. Rousset

2. Baudoz, P. et al. 2009, AO4ELT conf., Eds: Y. Clénet, J.M. Conan, T. Fusco, G. Rousset

3. Korkiakoski, V., Verinaud, C. 2009, AO4ELT conf., Eds: Y. Clénet, J.M. Conan, T. Fusco, G. Rousset

4. Martinez, P., Aller-Carpentier, E., Kasper, M., submitted to A\&AL, 2009

5. Martinez, P. et al., The Messenger 137, 2009, 18-23

6. Mordasini, C., Alibert, Y., Benz, W., A\&A 501, 2009, 1139-1160

7. Mugnier, L. M. et al., OExpr 22, 2008, 18406- 